\title{
LAS COLECCIONES MUSICALES DE LAS BIBLIOTECAS PÚBLICAS DE LA COMUNIDAD DE MADRID EN LA CAPITAL: APROXIMACIÓN TIPOLÓGICA Y ESTADÍSTICA
}

\author{
Esther Burgos Bordonau* \\ Facultad de Ciencias de la Documentación. Universidad Complutense de Madrid. \\ José Luis Palacios Gómez,** \\ Universidad Autónoma de Madrid
}

\begin{abstract}
Resumen: Estudio tipológico y estadístico de las colecciones musicales de las bibliotecas públicas de la Comunidad de Madrid en su capital. Para la realización de dicho estudio se hizo una consulta del catálogo general que permitió cuantificar los distintos soportes y los géneros musicales de los mismos. Los resultados del estudio revelan cuáles son los géneros musicales más demandados por los usuarios de estas bibliotecas y permiten un mayor conocimiento de sus gustos musicales. Los resultados también ponen de manifiesto que, aunque estas colecciones tienen un escaso volumen dentro del conjunto general de documentos de las bibliotecas, su uso en términos relativos es comparativamente muy superior al de las colecciones librarias.

Palabras clave: biblioteca pública; tipología documental; género musical; índice de rotación; índice de uso.
\end{abstract}

Title: MUSICAL COLLECTIONS FROM PUBLIC LIBRARIES OF COMUNIDAD DE MADRID IN ITS CAPITAL: TYPOLOGICAL AND STATISTICAL APPROACH.

Abstract: The aim of this work is to present a typological and statistical study of the musical collections in the public libraries of 'Comunidad de Madrid', specifically those in the capital. Reference was made to the main catalogue which gave insight into the different types of documents, as well as their different musical genres. The results of this study show which music styles are most in demand by the users of these libraries, and allows us to know their musical taste. These results also show that, although it's a small collection compared with the rest of the library's documents, its' use in relative terms is very high.

Keywords: public library; documental typology; musical genre; rotation index; use index.

\footnotetext{
* eburgos@pdi.ucm.es

** jl.palacios@uam.es
}

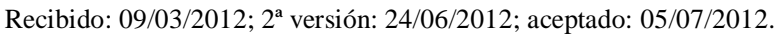

BURGOS BORDONAU, E. y PALACIOS GÓMEZ, J.L. Las colecciones musicales de las bibliotecas públicas de la Comunidad de Madrid en la capital: aproximación tipológica y estadística. Anales de Documentación, 2012, vol. 15, nº 2. ISSN: 1697-7904. http://dx.doi.org/10.6018/analesdoc.15.2.148991. 


\section{INTRODUCCIÓN}

Las colecciones $^{1}$ musicales que albergan las bibliotecas públicas han sido frecuentemente objeto de investigación en la mayor parte de los países que cuentan con alguna tradición biblioteconómica, como en Estados Unidos (Bradley, 1981; Fairtile y Burke, 2001), Canadá (Rose, 1987), Nueva Zelanda (Flury, 1990), Suecia (Holm, 2003), Hungría (Skaliczki, 1982), Holanda (Reeser, 1974), Dinamarca (Kjeldsen, 1987), Reino Unido (Fuidge; Willet, 2010; Hellen, 2000), Francia (Hausfater, 2002; Massault, 2002) o Japón (Poroila, 1989), y también en nuestro país (Bagüés, 2002, 2007; Fundación Germán Sánchez Ruipérez, 2003; Gosálvez, 1999; Iglesias, 2006, 2007; Montalt y otros, 2010).

Las colecciones musicales han sido estudiadas desde distintas perspectivas: desde el punto de vista del análisis documental y la clasificación (Díez, 1998, 2005; García, 1993, Fothergill y Butchart, 1992; Rodrigo, 2007; Gauchet, 2002), del marketing y las acciones divulgativas de las bibliotecas (Fenoll y Llueca, 2006; Herrera y Pérez, 2007) e incluso desde la perspectiva de la alfabetización informacional dentro del ámbito universitario (Cary y Sampsel, 2006). Las colecciones musicales de las bibliotecas también han sido estudiadas en aspectos tales como su gestión física y técnica (Cabezas, 2005; Gembero, 2005; Gómez, Hernández, Monterio y Vicente, 2008) o su lugar en las políticas culturales seguidas por las bibliotecas (Arranz, Carreño y Farré, 2008; Temes, 1993).

Paralelamente a la extensión y generalización de las colecciones musicales como un recurso más de las bibliotecas públicas, el personal de éstas se ha visto necesariamente obligado a formarse en las nuevas técnicas que la gestión de estos recursos precisaba en todos los órdenes. En consecuencia, la formación musical de los bibliotecarios ha sido también un tema investigado tanto en el ámbito de la biblioteca pública (Massault, 2002; Merlo, 1999) como en el de los centros de enseñanza musical (Lasarte, 2009; Lasarte y Arrué, 2000; Merlo, 1996). También existen estudios sobre el perfil de los profesionales de la documentación musical (Giráldez, 2005) y sobre la vinculación entre formación musical y la aplicación de las nuevas tecnologías en la biblioteca (Hunter, 2009; Plaza y Cuende, 1999).

Otros trabajos sobre colecciones musicales son aquellos dedicados específicamente a las fonotecas (Ambrosio, 1988, 1989; Estanyol y otros, 1998; Gallego, 1991; Miranda, 1990; Ruiz, 1985) o a las formas singulares del documento musical, como, por ejemplo, los discos de pizarra (Arévalo, 2000) o los cilindros de cera (Ranera y Crespo, 2010).

Sin embargo, son muy escasos los trabajos sobre la tipología del documento musical. La mayor parte de las veces, las referencias a esta cuestión en la literatura se solventan con una breve descripción de los géneros musicales pertinentes a efectos de catalogación. Y cuando se aborda el tema explícitamente, en algunos de los pocos estudios que lo tratan con el suficiente rigor y extensión (Torres, 1991, 1995, 2000; Burgos y Petrescu, 2011), la perspectiva es sobre todo cualitativa, atendiendo a las clases y características de los distintos tipos documentales, pero sin incidir apenas en el aspecto cuantitativo, es decir, en 
la relación entre tipos de documentos musicales y cantidad de los mismos. Es por eso que puede parecer especialmente oportuno dar algún paso en el camino de sustanciar con datos las colecciones musicales en las bibliotecas públicas, mostrando su tipología y las existencias de cada tipo. Ello proporcionaría una información biblioteconómica sin duda relevante, que concretaría la verdadera dimensión que los documentos musicales poseen en el seno de estas instituciones, su importancia relativa. Y si se añade a esta información el efectivo grado de uso que estas colecciones tienen, obtendremos una imagen completa de su auténtico peso sociocultural en la actualidad.

Con este trabajo queremos precisamente llevar a cabo una aproximación tipológica y estadística a las colecciones musicales de la red de bibliotecas públicas de la Comunidad de Madrid en su capital, poniendo de relieve cuáles y cuántas son esas colecciones, la dinámica de su gestión y la intensidad de la utilización que de ellos hacen los usuarios de estas bibliotecas, ofreciendo así una imagen fiel de su importancia relativa y su utilidad para los ciudadanos, proporcionando un indicador de su función sociocultural.

\section{PROCEDIMIENTO}

2.1. La red de bibliotecas públicas de la Comunidad de Madrid está formada por dieciséis bibliotecas, todas las cuales custodian una parte de las colecciones musicales objeto de nuestro estudio. La relación de estas bibliotecas, con su nombre y el distrito municipal en el que están ubicadas, es la siguiente:

- Acuña (Moncloa - Aravaca)

- Canillejas (San Blas)

- "Luis Rosales" (Carabanchel)

- Central (Chamberí)

- "Pedro Salinas" (Centro)

- "Rafael Alberti” (Fuencarral - El Pardo)

- Hortaleza (Hortaleza)

- “Antonio Mingote" (La Latina)

- Moratalaz (Moratalaz)

- Retiro (Retiro)

- "Ruiz Egea" (Chamberí)

- "Manuel Alvar" (Salamanca)

- “José Hierro" (Usera)

- Vallecas (Puente de Vallecas)

- "Luis Martín Santos" (Villa de Vallecas)

- "María Moliner" (Villaverde)

Solamente nueve de las bibliotecas ofrecen a sus usuarios puestos para audición o visionado de documentos.

2.2. Se procedió a realizar una explotación del catálogo de la Biblioteca Central (Chamberí), donde existe un registro general de las colecciones de todo tipo de las 16 
bibliotecas de la Comunidad de Madrid en la capital, usando el gestor de bibliotecas Absys Net, en diciembre de 2011. La explotación de esa base de datos se llevó a cabo sobre los siguientes criterios informativos:

- Número de soportes musicales por biblioteca en 2010

- Número de soportes musicales por biblioteca y tipo de soporte en 2010

- Número de soportes musicales por biblioteca y género musical en 2010

- Número de préstamos realizados, por biblioteca, tipo de soporte y género musical en 2010

2.3. La tipología de los documentos musicales de esta red de bibliotecas distingue los siguientes soportes: Disco Compacto (CD), Disco Compacto Read Only Memory (CD ROM), Disco Versátil Digital (DVD), Sistema de Vídeo Doméstico (VHS) y Disco BlueRay. Existen también documentos musicales en soporte DVD Rom, cassette y disco de vinilo, pero no los hemos considerado en nuestro estudio, los dos primeros debido a su escaso volumen (sólo cuatro ejemplares) y el último debido a que todos los ejemplares en este soporte están ya fuera de circulación.

2.4. El sistema de registro y catalogación de esta red de bibliotecas distingue los siguientes géneros musicales: música clásica, pop/rock, música popular (incluye el flamenco, la música étnica, música indie y géneros semejantes), jazz (incluye también soul, góspel y asimilados), bandas sonoras de películas, música escénica (incluye el ballet, la ópera y la zarzuela) y música new age.

\section{RESULTADOS}

La consulta del catálogo de la Biblioteca Central de esta red de bibliotecas en lo que se refiere a sus colecciones musicales arroja los resultados que se muestran en las Tablas I, II y III y en la Figura 1. 


\begin{tabular}{|c|c|c|c|c|c|c|c|c|}
\hline \multirow[b]{2}{*}{ BIBLIOTECA } & \multicolumn{8}{|c|}{ GÉNEROS MUSICALES } \\
\hline & $\begin{array}{l}\text { música } \\
\text { clásica }\end{array}$ & $\begin{array}{l}\text { pop/ } \\
\text { rock }\end{array}$ & popular & jazz & $\begin{array}{l}\text { bandas } \\
\text { sonoras }\end{array}$ & $\begin{array}{l}\text { música } \\
\text { escénica }\end{array}$ & new age & $\begin{array}{l}\text { Total } \\
\text { géneros }\end{array}$ \\
\hline ACUÑA & 288 & 667 & 268 & 156 & 145 & 205 & 62 & 1791 \\
\hline CANILLEJAS & 198 & 629 & 254 & 132 & 76 & 86 & 25 & 1400 \\
\hline CARABANCHEL & 433 & 1747 & 535 & 234 & 182 & 277 & 80 & 3488 \\
\hline CENTRAL & 568 & 1041 & 543 & 233 & 154 & 307 & 86 & 2932 \\
\hline CENTRO & 953 & 2834 & 974 & 465 & 248 & 525 & 169 & 6168 \\
\hline FUENCARRAL & 939 & 3770 & 893 & 470 & 371 & 436 & 172 & 7051 \\
\hline HORTALEZA & 78 & 535 & 211 & 88 & 63 & 53 & 27 & 1055 \\
\hline LATINA & 788 & 2514 & 738 & 389 & 238 & 428 & 154 & 5249 \\
\hline M. ALVAR & 512 & 1997 & 940 & 206 & 73 & 352 & 51 & 4131 \\
\hline MORATALAZ & 412 & 1396 & 478 & 250 & 258 & 206 & 99 & 3099 \\
\hline RETIRO & 1123 & 2565 & 903 & 400 & 318 & 500 & 152 & 5961 \\
\hline RUIZ EGEA & 958 & 2227 & 936 & 814 & 347 & 390 & 153 & 5825 \\
\hline USERA & 781 & 2731 & 1238 & 387 & 324 & 360 & 147 & 5968 \\
\hline VALLECAS & 944 & 3894 & 1108 & 537 & 418 & 406 & 173 & 7480 \\
\hline V. VALLECAS & 533 & 2394 & 814 & 404 & 320 & 253 & 121 & 4839 \\
\hline VILLAVERDE & 475 & 1994 & 469 & 259 & - & 309 & 88 & 3594 \\
\hline TOTALES & 9983 & 32935 & 11302 & 5424 & 3535 & 5093 & 1759 & 70031 \\
\hline
\end{tabular}

Tabla I. Colecciones musicales en soporte CD en 2010, por género musical. 


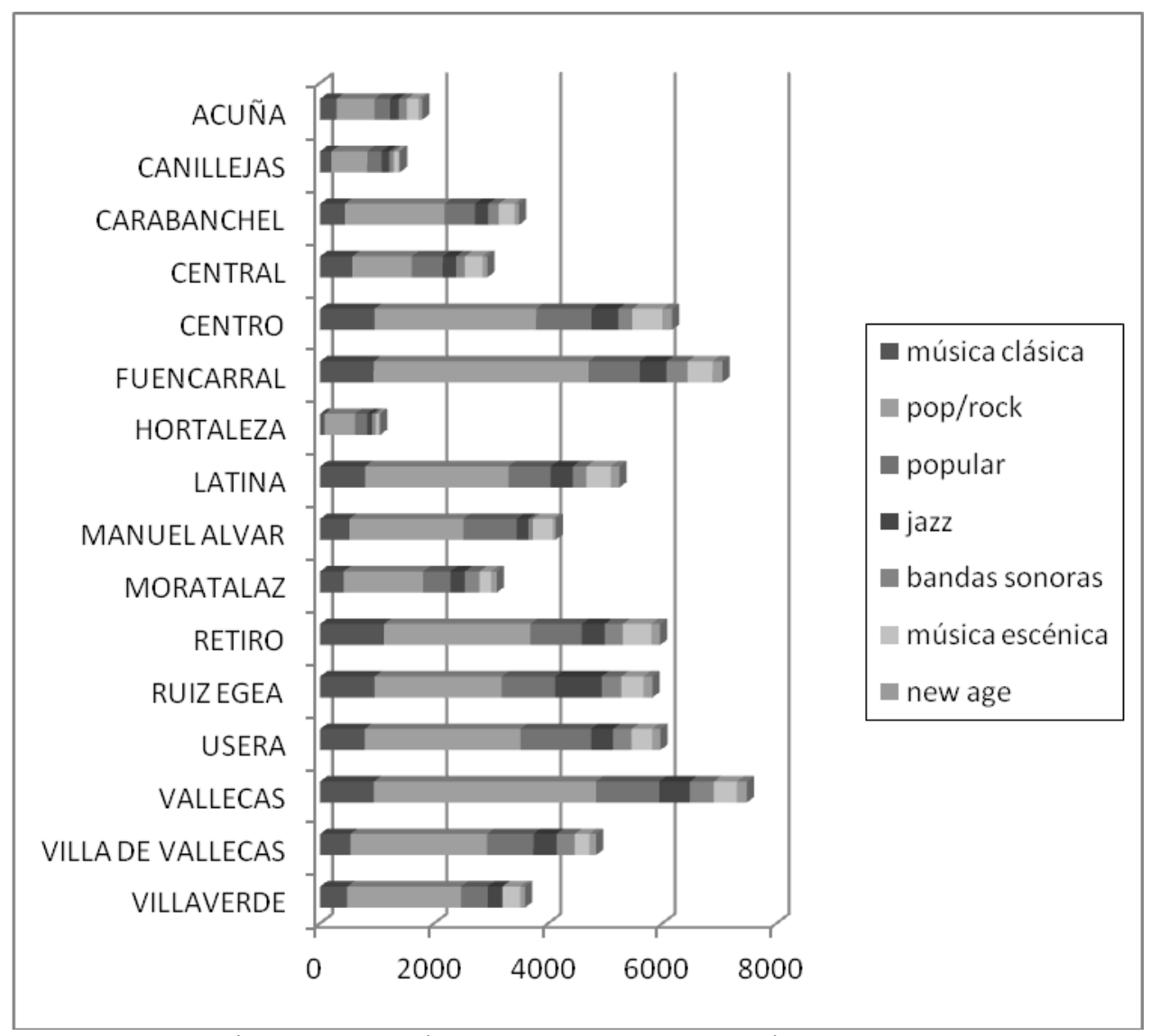

Figura 1. CD’s de todos los géneros musicales en circulación en 2010, por biblioteca. 


\begin{tabular}{|l|l|l|l|l|l|l|l|l|}
\hline & \multicolumn{7}{|}{} & \multicolumn{7}{|c|}{ GÉNEROS MUSICALES } \\
\cline { 2 - 8 } & $\begin{array}{l}\text { música } \\
\text { clásica }\end{array}$ & $\begin{array}{l}\text { pop/ } \\
\text { rock }\end{array}$ & popular & jazz & $\begin{array}{l}\text { bandas } \\
\text { sonoras }\end{array}$ & $\begin{array}{l}\text { música } \\
\text { escénica }\end{array}$ & new age & $\begin{array}{l}\text { Total } \\
\text { géneros }\end{array}$ \\
\hline ACUÑA & - & 7 & 1 & 1 & - & 1 & - & 10 \\
\hline CANILLEJAS & - & 1 & - & - & - & - & - & 1 \\
\hline CARABANCHEL & - & - & - & - & - & - & - & 0 \\
\hline CENTRAL & - & 9 & 1 & 1 & 1 & 1 & - & 13 \\
\hline CENTRO & - & - & 1 & & & 1 & - & 2 \\
\hline FUENCARRAL & - & 1 & - & - & - & - & - & 1 \\
\hline HORTALEZA & - & 4 & - & - & 1 & 2 & - & 7 \\
\hline LATINA & - & 3 & - & - & - & - & - & 3 \\
\hline M. ALVAR & - & 2 & 1 & & 1 & - & - & 4 \\
\hline MORATALAZ & - & 9 & 7 & 2 & 4 & 3 & - & 25 \\
\hline RETIRO & - & 1 & 1 & - & - & - & - & 2 \\
\hline RUIZ EGEA & - & 1 & 1 & 2 & 1 & - & 1 & 6 \\
\hline USERA & - & 3 & - & 1 & - & - & 1 & 5 \\
\hline VALLECAS & - & 2 & - & 1 & - & 1 & - & 4 \\
\hline V. VALLECAS & - & - & - & - & - & - & - & 0 \\
\hline VILLAVERDE & - & 4 & - & - & - & - & - & 4 \\
\hline TOTALES & - & $\mathbf{4 7}$ & $\mathbf{1 3}$ & $\mathbf{8}$ & $\mathbf{8}$ & $\mathbf{9}$ & $\mathbf{2}$ & $\mathbf{8 7}$ \\
\hline
\end{tabular}

Tabla II. Colecciones musicales en soporte CD ROM en 2010, por género musical. 


\begin{tabular}{|c|c|c|c|c|c|c|c|c|}
\hline \multirow[b]{2}{*}{ BIBLIOTECA } & \multicolumn{8}{|c|}{ GÉNEROS MUSICALES } \\
\hline & $\begin{array}{l}\text { música } \\
\text { clásica }\end{array}$ & $\begin{array}{l}\text { pop/ } \\
\text { rock }\end{array}$ & popular & jazz & $\begin{array}{l}\text { bandas } \\
\text { sonoras }\end{array}$ & $\begin{array}{l}\text { música } \\
\text { escénica }\end{array}$ & new age & $\begin{array}{l}\text { Todos los } \\
\text { géneros }\end{array}$ \\
\hline ACUÑA & - & 3 & - & - & - & - & - & 3 \\
\hline CANILLEJAS & - & 3 & 1 & 1 & - & - & - & 5 \\
\hline CARABANCHEL & - & 2 & - & 1 & - & - & - & 3 \\
\hline CENTRAL & - & 4 & 3 & - & 1 & - & - & 8 \\
\hline CENTRO & - & 1 & - & - & - & - & - & 1 \\
\hline FUENCARRAL & - & 3 & 1 & - & 1 & 1 & & 6 \\
\hline HORTALEZA & - & 1 & - & - & - & - & - & 1 \\
\hline LATINA & - & 3 & 1 & - & - & - & - & 4 \\
\hline M. ALVAR & - & 6 & 1 & - & - & - & - & 7 \\
\hline MORATALAZ & - & 1 & 3 & - & - & 2 & - & 6 \\
\hline RETIRO & - & 2 & 1 & - & - & - & - & 3 \\
\hline RUIZ EGEA & - & 12 & 5 & 6 & 1 & 3 & 1 & 28 \\
\hline USERA & - & 9 & 2 & - & - & - & - & 11 \\
\hline VALLECAS & - & 1 & 2 & & - & 1 & - & 4 \\
\hline V. VALLECAS & - & 2 & 1 & 1 & - & 1 & - & 5 \\
\hline VILLAVERDE & - & 2 & - & - & - & 1 & - & 3 \\
\hline TOTALES & - & 55 & 21 & 9 & 3 & 8 & 1 & 95 \\
\hline
\end{tabular}

Tabla III. Colecciones musicales en soporte DVD en 2010, por género musical.

Como vemos, el grueso de las colecciones musicales de esta red de bibliotecas está constituido por discos compactos (CD's), mientras que los CD ROM's y los DVD's con contenido estrictamente musical sólo alcanzan 87 y 95 ejemplares, respectivamente. Las colecciones musicales relativos a soportes VHS y Blue-Ray los hemos soslayado debido a su escaso volumen, pues para el conjunto de las dieciséis bibliotecas solamente alcanzan unos cuantos ejemplares que no merece la pena someter a explotación estadística.

Si nos centramos en las colecciones musicales en soporte $C D$, que alcanzan para el conjunto de las bibliotecas de la red un total de 70.031 unidades, la distribución por género musical es la que puede verse en la tabla 4 y en la Figura 2, donde puede apreciarse el peso relativo de cada género sobre el total de estas colecciones. Como vemos, el mayor peso relativo lo ofrece el género musical Pop/Rock, que constituye casi la mitad de las existencias del fondo en soporte CD (47,03\%). El segundo y el tercer lugar lo ocupan los géneros Música Popular y Música Clásica (16,14\% y 14,26\%, respectivamente). En las colecciones musicales en soporte CD ROM y DVD los géneros con mayor peso relativo los presentan también la música Pop/Rock (con 54,02\% y 
57,89\%, respectivamente) y la Música Popular (14,94\% y 22,11\%), pero la Música Clásica no cuenta con presencia alguna en estos soportes (véanse Tablas V y VI).

\begin{tabular}{|l|l|l|l|l|l|l|l|}
\hline $\begin{array}{l}\text { Música } \\
\text { clásica }\end{array}$ & $\begin{array}{l}\text { Pop/ } \\
\text { Rock }\end{array}$ & Popular & Jazz & $\begin{array}{l}\text { Bandas } \\
\text { sonoras }\end{array}$ & $\begin{array}{l}\text { Música } \\
\text { escénica }\end{array}$ & New age & $\begin{array}{l}\text { Todos los } \\
\text { géneros }\end{array}$ \\
\hline 9983 & 32935 & 11302 & 5424 & 3535 & 5093 & 1759 & 70031 \\
\hline $14,26 \%$ & $47,03 \%$ & $16,14 \%$ & $7,75 \%$ & $5,05 \%$ & $7,27 \%$ & $2,51 \%$ & $100,00 \%$ \\
\hline
\end{tabular}

Tabla IV. Distribución por género de las colecciones musicales en soporte CD del conjunto de bibliotecas.

\begin{tabular}{|l|l|l|l|l|l|l|l|}
\hline $\begin{array}{l}\text { Música } \\
\text { clásica }\end{array}$ & $\begin{array}{l}\text { Pop/ } \\
\text { Rock }\end{array}$ & Popular & Jazz & $\begin{array}{l}\text { Bandas } \\
\text { sonoras }\end{array}$ & $\begin{array}{l}\text { Música } \\
\text { escénica }\end{array}$ & New age & $\begin{array}{l}\text { Todos los } \\
\text { géneros }\end{array}$ \\
\hline 0 & 47 & 13 & 8 & 8 & 9 & 2 & 87 \\
\hline $0,00 \%$ & $54,02 \%$ & $14,94 \%$ & $9,20 \%$ & $9,20 \%$ & $10,34 \%$ & $2,30 \%$ & $100,00 \%$ \\
\hline
\end{tabular}

Tabla V. Distribución por género de las colecciones musicales en soporte CD ROM del conjunto de bibliotecas.

\begin{tabular}{|l|l|l|l|l|l|l|l|}
\hline $\begin{array}{l}\text { Música } \\
\text { clásica }\end{array}$ & $\begin{array}{l}\text { Pop/ } \\
\text { Rock }\end{array}$ & Popular & Jazz & $\begin{array}{l}\text { Bandas } \\
\text { sonoras }\end{array}$ & $\begin{array}{l}\text { Música } \\
\text { escénica }\end{array}$ & New age & $\begin{array}{l}\text { Todos los } \\
\text { géneros }\end{array}$ \\
\hline 0 & 55 & 21 & 9 & 3 & 8 & 1 & 95 \\
\hline $0,00 \%$ & $57,89 \%$ & $22,11 \%$ & $9,47 \%$ & $3,16 \%$ & $8,42 \%$ & $1,05 \%$ & $100,00 \%$ \\
\hline
\end{tabular}

Tabla VI. Distribución por género de las colecciones musicales en soporte DVD del conjunto de bibliotecas.

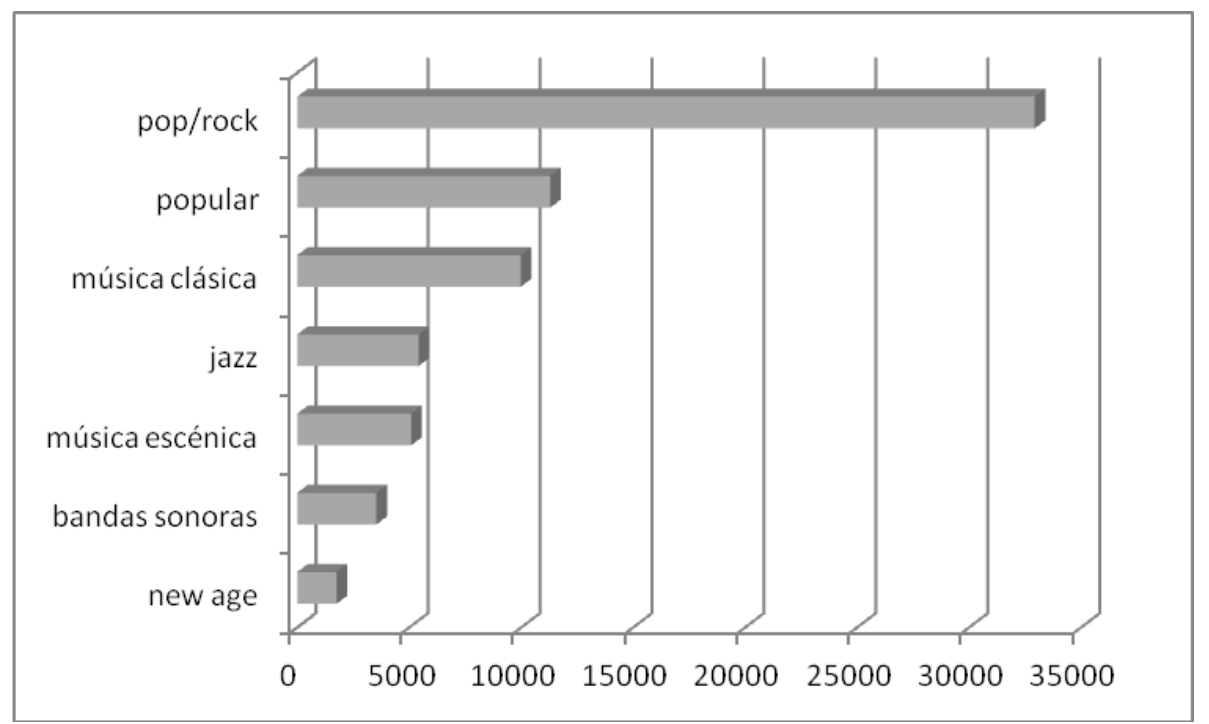

Figura 2. Número de CD's de los distintos géneros musicales en el conjunto de bibliotecas en 2010. 
Si ahora consideramos los datos referidos a los préstamos de las colecciones musicales efectuados en las bibliotecas de esta red en 2010, el resultado es el que se muestra en la Tabla VII.

\begin{tabular}{|c|c|c|c|c|c|c|c|c|}
\hline \multirow[b]{2}{*}{ BIBLIOTECA } & \multicolumn{8}{|c|}{ GÉNEROS MUSICALES } \\
\hline & $\begin{array}{l}\text { música } \\
\text { clásica }\end{array}$ & $\begin{array}{l}\text { pop/ } \\
\text { rock }\end{array}$ & popular & jazz & $\begin{array}{l}\text { bandas } \\
\text { sonoras }\end{array}$ & $\begin{array}{l}\text { música } \\
\text { escénica }\end{array}$ & new age & $\begin{array}{l}\text { Total } \\
\text { géneros }\end{array}$ \\
\hline ACUÑA & 873 & 2622 & 970 & 1032 & 630 & 397 & 181 & 6705 \\
\hline CANILLEJAS & 91 & 265 & 130 & 44 & 7 & 29 & 14 & 580 \\
\hline CARABANCHEL & 479 & 3832 & 808 & 708 & 525 & 168 & 367 & 6887 \\
\hline CENTRAL & 479 & 768 & 476 & 363 & 159 & 177 & 120 & 2542 \\
\hline CENTRO & 2760 & 7528 & 2230 & 1761 & 1268 & 1223 & 640 & 17410 \\
\hline FUENCARRAL & 1927 & 8699 & 1717 & 1277 & 786 & 516 & 582 & 15504 \\
\hline HORTALEZA & 31 & 285 & 122 & 23 & 23 & 4 & 14 & 502 \\
\hline LATINA & 1605 & 7336 & 1740 & 1166 & 687 & 499 & 741 & 13774 \\
\hline M. ALVAR & 1928 & 4143 & 1924 & 1058 & 300 & 657 & 424 & 10434 \\
\hline MORATALAZ & 462 & 2631 & 466 & 561 & 509 & 90 & 85 & 4804 \\
\hline RETIRO & 3467 & 9044 & 2112 & 2264 & 1134 & 821 & 604 & 19446 \\
\hline RUIZ EGEA & 2535 & 11996 & 2668 & 3653 & 1401 & 806 & 1048 & 24107 \\
\hline USERA & 1837 & 8898 & 2909 & 1397 & 1203 & 508 & 724 & 17476 \\
\hline VALLECAS & 1064 & 7219 & 1686 & 811 & 459 & 275 & 306 & 11820 \\
\hline V. VALLECAS & 684 & 7745 & 1499 & 817 & 961 & 165 & 506 & 12377 \\
\hline VILLAVERDE & 771 & 6966 & 1550 & 1036 & - & 374 & 280 & 10977 \\
\hline TOTALES & 20993 & 89977 & 23007 & 17971 & 10052 & 6709 & 6636 & 175345 \\
\hline
\end{tabular}

Tabla VII. Préstamos de las colecciones musicales en todos los soportes en 2010, por género.

Como puede verse, los géneros musicales que presentan una mayor cantidad de unidades demandadas por los usuarios de estas bibliotecas son la música Pop/Rock (89.977), la Música Popular (23.007) y la Música Clásica (20.993).

Si tenemos en cuenta que las colecciones musicales en soporte CD ROM y DVD representan solamente el $0,26 \%$ del total de soportes musicales y que los otros soportes musicales (VHS y disco Blue-Ray) tienen un peso aún menor sobre el total, se puede concluir que la práctica totalidad de los préstamos de documentos musicales se presentan en soporte CD. En consecuencia, no parece en modo alguno muy impreciso identificar en términos operativos los datos relativos a los préstamos de CD's con los datos relativos a los préstamos de las colecciones musicales de esta red de bibliotecas. Si aceptamos esto, podemos relacionar con un indicador apropiado el volumen de colecciones musicales en este soporte con el volumen de préstamos total de toda clase de documentos para dar 
noticia de la intensidad de utilización de las colecciones musicales por los usuarios de estas bibliotecas. El volumen total de préstamos del conjunto de bibliotecas de esta red fue en 2010 de aproximadamente $2.548 .000^{2}$. Un indicador válido de uso de sus colecciones musicales puede ser el siguiente:

$$
I_{u}=\frac{V_{p M}}{V_{p T}}=\frac{175.345}{2.548 .000}=0,0688
$$

donde $V_{P M}$ es el volumen de préstamos de documentos musicales y $V_{P T}$ es el volumen de préstamos totales de cualquier tipo de documento de los colecciones de las bibliotecas. Por consiguiente, el indicador de uso $I_{u}$ es 0,0688 , que significa simplemente que en torno a un $7 \%$ de los documentos que se prestan en esta red de bibliotecas son unidades de sus colecciones musicales.

Podemos aplicar la misma lógica estadística para expresar el uso de los documentos musicales de los distintos géneros, empleando el siguiente índice:

$$
I_{u G}=\frac{V_{p M G}}{V_{p M T}}
$$

donde $V_{p M G}$ es el volumen de préstamos de documentos musicales de un determinado género y $V_{p T M}$ es el volumen total de préstamos de documentos musicales. Calculando el índice para los siete géneros musicales con los que trabaja esta red de bibliotecas obtenemos unos resultados como los que se muestran en la Tabla VIII.

\begin{tabular}{|l|l|l|l|l|l|l|l|}
\hline Géneros & $\begin{array}{l}\text { música } \\
\text { clásica }\end{array}$ & $\begin{array}{l}\text { pop/ } \\
\text { rock }\end{array}$ & popular & jazz & $\begin{array}{l}\text { bandas } \\
\text { sonoras }\end{array}$ & $\begin{array}{l}\text { música } \\
\text { escénica }\end{array}$ & new age \\
\hline$I_{u G}$ & 0,1197 & 0,5131 & 0,1312 & 0,1025 & 0,0573 & 0,0383 & 0,0378 \\
\hline
\end{tabular}

Tabla VIII. Índices de uso de las colecciones musicales por género.

Para expresar los índices $I_{u G}$ en forma porcentual y facilitar su interpretación, basta con multiplicarlos por cien y observarlos como si fuesen una tasa. Como puede verse (Figura 3), los géneros musicales que suscitan en los usuarios de estas bibliotecas una mayor demanda de préstamo son la música Pop/Rock (51,31\% del total de préstamos), la Música Popular $(13,12 \%)$ y la Música Clásica $(11,97 \%)$, en coincidencia, naturalmente, con el orden que ya vimos en las cifras brutas de préstamos por género. 


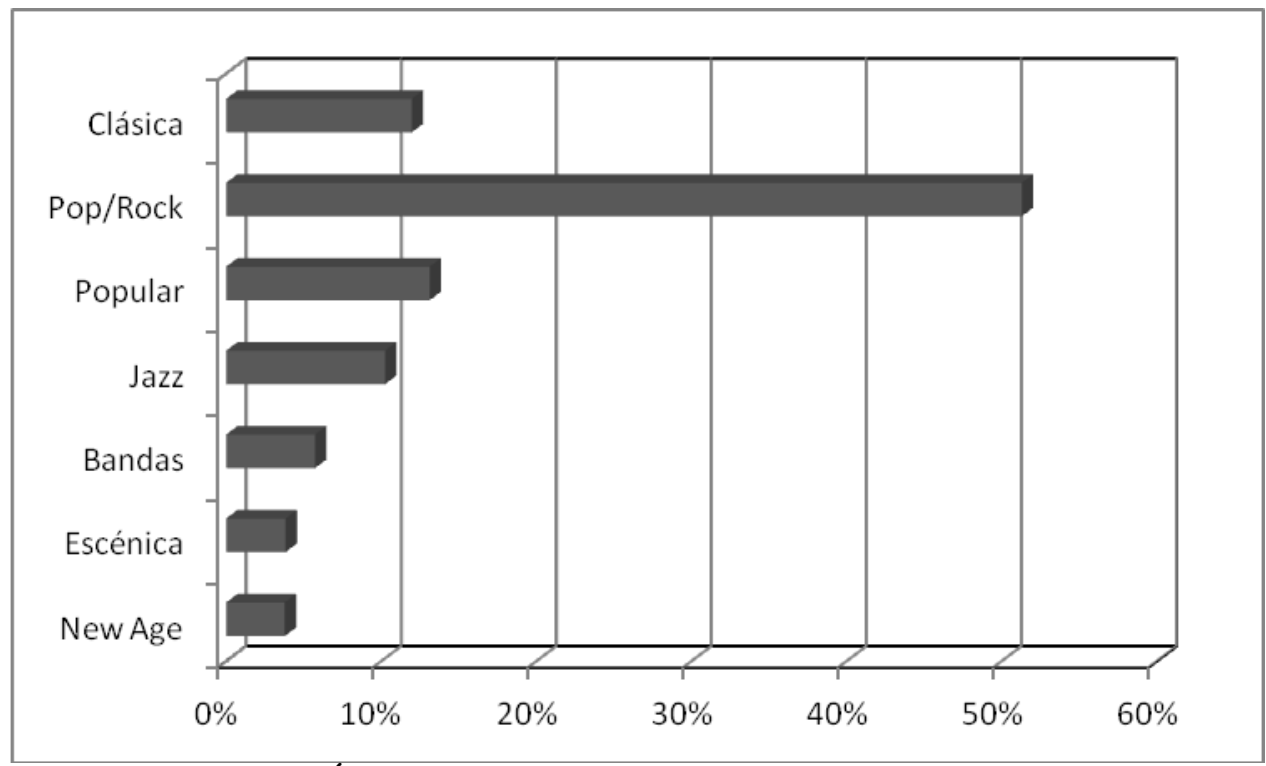

Figura 3. Índices de uso de los distintos géneros musicales (en \%).

Complementariamente, también podemos matizar la información relativa al uso de las colecciones musicales con el conocido índice de rotación (Setién, 1998), usado para cualquier tipo de fondo documental, que es el cociente entre el número de documentos prestados y el número de documentos que contiene un fondo. Aplicado a los colecciones musicales de esta red de bibliotecas y teniendo en cuenta lo apuntado respecto a la identificación operativa de volumen de préstamos con volumen de préstamos de CD's, su índice de rotación de los colecciones musicales es $I_{R M}=175.345 / 70213=2,49$, cuyo significado es que, en promedio, cada documento de estas colecciones musicales se presta, aproximadamente 2,5 veces (frente a 1,56 para el total de los fondos de esta red de bibliotecas $\left.^{3}\right)$. Si aplicamos el mismo índice parcialmente, para cada género musical $\left(I_{R M G}\right)$, el resultado es el que se muestra en la Tabla IX.

\begin{tabular}{|l|l|l|l|l|l|l|l|}
\hline Géneros & $\begin{array}{l}\text { música } \\
\text { clásica }\end{array}$ & $\begin{array}{l}\text { pop/ } \\
\text { rock }\end{array}$ & popular & jazz & $\begin{array}{l}\text { bandas } \\
\text { sonoras }\end{array}$ & $\begin{array}{l}\text { música } \\
\text { escénica }\end{array}$ & new age \\
\hline$I_{R M G}$ & 2,10 & 2,73 & 2,04 & 3,31 & 2,84 & 1,32 & 3,77 \\
\hline
\end{tabular}

Tabla IX. Índice de rotación de las colecciones musicales por género.

Como puede apreciarse, los mayores índices de rotación los presentan las colecciones musicales de los géneros new age $(3,77)$ y jazz $(3,31)$. De manera que, si bien las colecciones musicales de los géneros pop/rock, música popular y música clásica son, como hemos visto, los más demandados por los usuarios de las bibliotecas en términos absolutos, su utilidad relativa, medida con el índice de rotación $(2,73,2,04$ y 2,10, 
respectivamente), es notablemente inferior a las colecciones musicales de los otros dos géneros antes señalados. En la Figura 4 se muestran los índices de uso por género (transformado en escala 0-10 para mejor compararlo) y de rotación por género, apreciándose el contraste señalado.

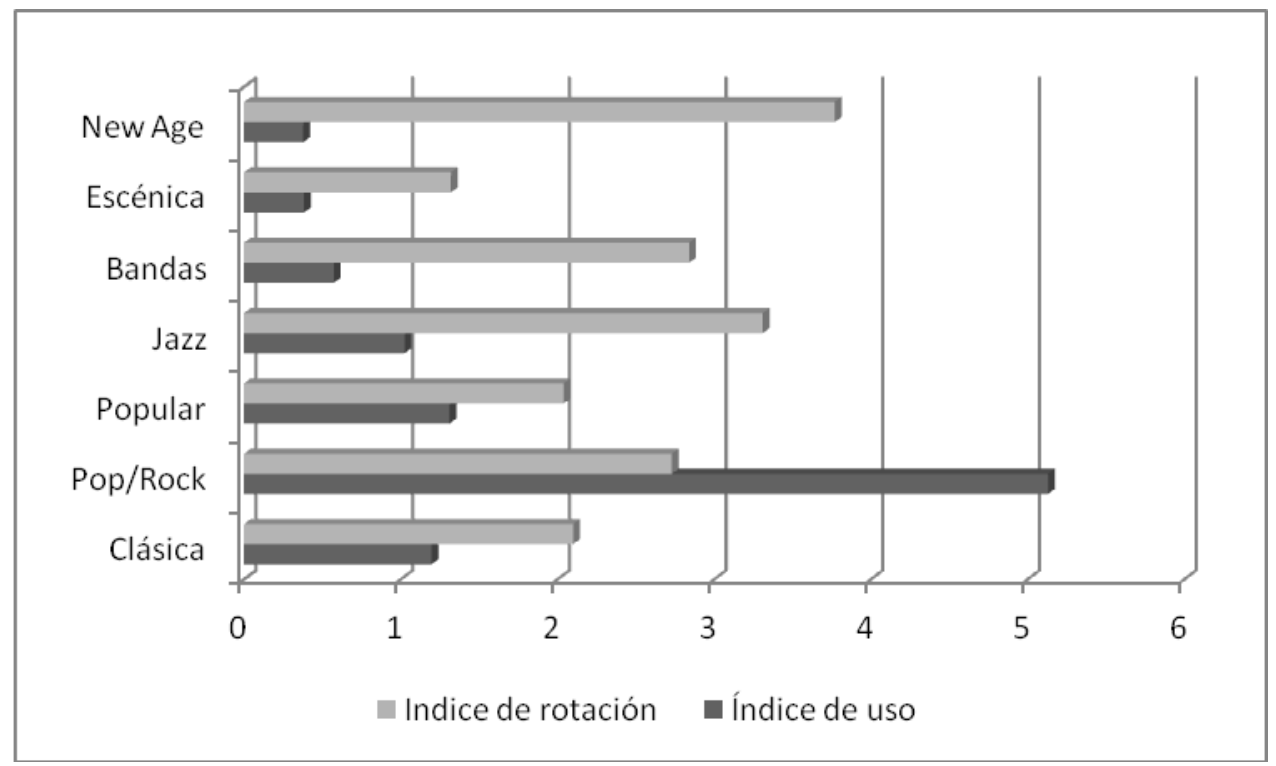

Figura 4. Comparación de los índices de uso (trasformado) y de rotación de los distintos géneros musicales.

Finalmente, también merece la pena apuntar aquí que, además de las colecciones musicales que se han reseñado más arriba, la red de bibliotecas públicas de la Comunidad de Madrid en la capital posee, entre los documentos musicales que alberga, un pequeño número de partituras musicales, bajo la rúbrica de "música impresa". Sin embargo, su muy exiguo volumen (15 ejemplares, en total) hace que no merezca la pena someterlos a ninguna clase de explotación estadística.

\section{CONCLUSIONES}

En este estudio se ha puesto de relieve cuál es el volumen de las colecciones musicales de las bibliotecas públicas de la Comunidad de Madrid en su capital y la tipología de los soportes de los mismos. Igualmente se ha determinado cuáles son los géneros musicales en los que se distribuyen estas colecciones y el peso que tiene cada una dentro del conjunto. Ello ha permitido caracterizar desde el punto de vista físico y clasificatorio las colecciones musicales de esta importante red de bibliotecas públicas de Madrid. Esta clase de información no suele hallarse en la literatura biblioteconómica, especialmente en lo que se refiere al detalle tipológico, que muy raramente distingue además los géneros musicales de los distintos soportes. Los datos de este estudio permiten matizar las 
características de estas colecciones de un modo pormenorizado, de resultas del cual se puede tener una idea precisa de su composición y constituyen un avance con respecto a los habituales patrones bibliotecológicos descriptivos, en general, y evaluativos, en particular (Abad, 2005; Fuentes, 1999; Lancaster, 1996).

Por otra parte, nos hemos detenido en este trabajo en la cuestión de la utilización efectiva de las colecciones musicales por los usuarios de estas bibliotecas. Pensamos que este es un aspecto fundamental de la investigación de las colecciones bibliotecarias, pues da noticia de la verdadera utilidad social de este recurso cultural público, utilidad que sería puesta en cuestión si, siendo conocido y accesible el recurso, los ciudadanos declinasen usarlo (sin perjuicio de su posible valor intrínseco desde el punto de vista histórico, musicológico, archivístico u otro cualquiera). Nuestro análisis de este aspecto de las colecciones musicales se ha centrado en tres indicadores: los índices de uso general, de uso por género musical y de rotación. El primero de ellos pone de relieve que el $7 \%$ aproximadamente de los documentos que se prestan en la red de bibliotecas analizada son soportes musicales (fundamentalmente CD’s); el segundo, que los géneros musicales más demandados son el pop/rock, la música popular y la música clásica (por este orden); el tercero, en fin, que el promedio de uso de estas colecciones es de 2,5 veces, aproximadamente, por documento, y que los géneros musicales más usados de estos documentos son el new age y el jazz, poniendo de manifiesto que, aunque en términos absolutos son otros los géneros más demandados, los géneros que presentan un mayor rendimiento neto son los dos antes señalados. Con respecto al segundo indicador, el de los géneros musicales más demandados en términos absolutos, merece la pena hacer notar aquí que los resultados que hemos obtenido para esta red de bibliotecas pueden contrastarse con los géneros musicales más demandados por la población en general: en la encuesta del Ministerio de Cultura de "Hábitos y Prácticas Culturales" (Ministerio de Cultura, 2011) se muestra que del total de CD's adquiridos por los ciudadanos en el último trimestre antes de la encuesta en 2010 el 52,41\% era del género rock/pop, el $11,63 \%$ era del género música popular y el 5,30\% era del género música clásica, lo cual pone de manifiesto el paralelismo entre consumo de la población en general y consumo de los usuarios de estas bibliotecas en lo que se refiere a la música rock/pop y la música popular, pero no así en lo que se refiere a la música clásica, respecto de la cual la población de usuarios muestra una inclinación el doble de intensa que la población general.

Por otra parte, también hay que reparar en el hecho de que si bien las colecciones musicales de las Bibliotecas Públicas de la Comunidad de Madrid en su capital constituyen una parte minoritaria del conjunto de colecciones de toda índole que alberga esta red de bibliotecas (aproximadamente el $4 \%$ del fondo total), su índice de rotación es un $60 \%$ superior al índice de rotación general de todos los documentos (y un $82 \%$ superior al de todos los colecciones no musicales), lo cual nos ofrece una matización ponderada de su uso y, por consiguiente, de su utilidad relativa, que es la realmente relevante. 
En definitiva, una explotación estadística y tipológica del catálogo de las colecciones musicales, como la llevada a cabo aquí, permite percatarse de ciertos aspectos y matices biblioteconómicos y socioculturales que a nuestro juicio enriquecen muy considerablemente la visión de las colecciones de las bibliotecas públicas y su proyección social, proporcionando algunos de los parámetros necesarios con los que han de ser evaluadas globalmente como recursos culturales. Obviamente, abundar en la realización de estudios como el presente en otras bibliotecas u otras redes bibliotecarias contribuirá a aumentar y perfeccionar nuestro conocimiento sobre las características de las colecciones musicales públicos, su gestión y su utilidad para la comunidad.

\section{AGRADECIMIENTOS}

Los autores desean agradecer al personal de las Bibliotecas Públicas de la Comunidad de Madrid y muy especialmente al responsable de su Unidad Central, D. Carlos García Romeral, la colaboración prestada para la realización de este estudio.

\section{NOTAS}

${ }^{1}$ Aunque en este trabajo se emplea el término "colecciones", en buena parte de la bibliografía y de las fuentes consultadas se utiliza el término "fondos" para referirse a los elencos de este tipo de documentos.

${ }^{2}$ Comunicación personal del responsable de la Unidad Central de Bibliotecas de la Comunidad de Madrid (diciembre de 2011).

${ }^{3}$ Comunicación personal del responsable de la Unidad Central de Bibliotecas de la Comunidad de Madrid (diciembre de 2011).

\section{BIBLIOGRAFÍA}

ABAD, M.F. Evaluación de la calidad de los sistemas de información. Madrid: Síntesis, 2005.

AMBROSIO, A. Servicios de fonoteca en bibliotecas populares. Boletín de la ANABAD, 1988, vol. 38, nº 4, p. 371-381.

AMBROSIO, A. Servicio de fonoteca en bibliotecas. Una propuesta para bibliotecas pequeñas. Boletín de la Asociación Andaluza de Bibliotecarios, 1989, n 14, p. 31-40.

ARÉVALO, J. El fondo de los discos de pizarra de la Biblioteca General de Ciudad Real. 2000. [en línea] Disponible en: <http://biblioteca2.uclm.es/biblioteca/Ceclm/ websCECLM/discosradio/estudios/Julioarevalo.html>. [Consulta: 22 de febrero de 2012].

ARRANZ, J.; CARREÑO, O. y FARRÉ, F. Políticas culturales y biblioteca pública del siglo XXI. Conversaciones sobre algunos temas relevantes. Periférica, 2008, n⿳⺈ 9, . 41-59.

BAGÜÉS, J. Fondos musicales en Eresbil - Musikaren Euskal Artxiboa. Musiker, Cuadernos de Música, 2002, n 13, p. 221-252.

BAGÜÉS, J. Fondos musicales en bibliotecas y archivos de Euskal Herria. Biblid, 2007, $\mathrm{n}^{\mathrm{o}} 15, \mathrm{p} \cdot 271-295$. 
BRADLEY, C. Music collections in American libraries: a chronology. Detroit: Information Coordinators, 1981.

BURGOS, E. y PETRESCU, C. Tipology of the musical document: an approach to its study. Bulletin of the Transilvania University of Brasov, 2011, Series VIII: Art-Sport, vol. 4, (53), no 2, p. 31-38.

CABEZAS, E. La organización de archivos musicales: marco conceptual. Información, Cultura y Sociedad, 2005, nº 13, p. 81-99.

CARY, P. y SAMPSEL, L.J. Objetivos de formación en alfabetización informacional para estudiantes de música universitarios. Boletín DM, Aedom, 2006, año 10, p. 55-75.

DÍEZ, C. (coord. y dir). Los materiales especiales en las bibliotecas. Gijón: Trea, 1998.

DÍEZ, C. (coord. y dir). La catalogación de los materiales especiales. Gijón: Trea, 2005.

ESTANYOL, M.; CUENDE, M. y SUNYER, R. Fonoteca de la Biblioteca de Catalunya. Actas del $18^{\circ}$ Congreso de la Asociación Internacional de Bibliotecas Musicales, Archivos y Centros de Documentación. Madrid: Aedom, 1999, p. 297-307.

FAIRTILE, L.B. y BURKE, K.M. Music collections in American public libraries. Fontes Artis Musicae, 2001, vol. 48, nº 4, p. 327-341.

FENOLL, C. y LLUECA, C. Cincuenta ideas para sorprender desde la biblioteca pública. BID, 2006, $\mathrm{n}^{\circ}$ 17. [en línea]. Disponible en: 〈http://www.ub.edu/bid/17fenol1.htm>. [Consulta: 10 de diciembre de 2011].

FLURY, R. Music in public libraries in New Zealand and the sound and music centre. Fontes Artis Musicae, 1990, vol. 37, n 2, p. 172-176.

FOTHERHILL, R. y BUTCHART, I. Materiales no librarios en las bibliotecas: guía práctica. (3 ${ }^{a}$ ed.) Madrid: Fundación Germán Sánchez Ruipérez, 1992.

FUENTES, J.J. Evaluación de bibliotecas y centros de documentación e información. Gijón: Ediciones Trea, 1999.

FUIDGE, V. y WILLET, P. The role of the music specialist in music collections in English public libraries. Brio: Journal of the United Kingdom branch of the IAML, 2010, vol. 47, no 2, p. 37-46.

FUNDACIÓN GERMÁN SÁNCHEZ RUIPÉREZ. Las colecciones de las bibliotecas públicas en España. Informe de situación. 2003. [en línea]. Disponible en: <http://www.bibliotecaspublicas.info/colecbp/coleccion/CCAA/colecBP_asturias.pdf> [Consulta: 10 de enero de 2012].

GARCÍA, F. Materiales bibliográficos especiales: manual de ejercicios de catalogación. Gijón: Trea, 1993.

GALLEGO, M.P. La Fonoteca nacional. Boletín de la ANABAD, 1991, vol. 41, n 3-4, p. 529-535.

GAUCHET, I. La description de la musique. Bulletin des Bibliothèques de France, 2002, vol. 47, n' 2, p. 85-89.

GEMBERO, M. El patrimonio musical español y su gestión. Revista de Musicología, 2005, vol. 28, no 1, p. 135-181.

GIRÁLDEZ, A. Internet y educación musical. Barcelona: Ed. Graó, 2005.

GÓMEZ, P.J.; HERNÁNEZ, L.; MONTERO, J. y VICENTE, R. El archivo de los sonidos: la gestión de los fondos musicales. Salamanca: Acal, 2008. 
GOSÁLVEZ, J. Las colecciones musicales de la Biblioteca Nacional y del Real Conservatorio de Madrid. Actas del $18^{\circ}$ Congreso de la Asociación Internacional de Bibliotecas Musicales, Archivos y Centros de Documentación. Madrid: Aedom, 1999, p. 281-291.

HAUSFATER, D. Une cartographie des fonds musicaux en France. Bulletin des Bibliothèques de France, 2002, vol. 47, nº 2, p. 23-27.

HELLEN, R. A good thing: public music libraries in the U.K. Crescendo: Bulletin of the International Association of Music Libraries (New Zealand Branch), 2000, $\mathrm{n}^{\circ}$ 55, $\mathrm{p}$. 12-15.

HERRERA, J.L. y PÉREZ, M. Cultura y marketing en las bibliotecas públicas españolas: actividades, iniciativas y recursos. El profesional de la información, 2007, vol. 16, $\mathrm{n}^{\mathrm{o}}$ 1, p. 62-76.

HOLM, A. Music collections in research libraries in Sweden: an overview of major collections in public institutions. En: Lönn, Anders (colab.) Inte bara katalogregler: Festkrift till Anders Lönn. Stockholm: Statens Musikbibliotek, 2003.

HUNTER, D. Competencias básicas de los bibliotecarios musicales. Boletín DM, Aedom, 2009, año 13, p. 45-48.

IGLESIAS, N. Bibliotecas e investigación musical. Arbor, 2006, vol. 182, n 717, p. 5565.

IGLESIAS, N. La música en la Biblioteca Nacional de España. Boletín DM, Aedom, 2007, año 11, p. 14-43.

KJELDSEN, B. Public music libraries in Denmark. Fontes Artis Musicae, 1987, vol. 34, $\mathrm{n}^{\circ} 2-3$, p. 150-153.

LANCASTER, F.W. Evaluación de la Biblioteca. Madrid: Anabad, 1996.

LASARTE, A. Diez años de la biblioteca de la Escuela Municipal de Música y Danza de Donostia-San Sebastián. Boletín DM, Aedom, 2009, año 13, p. 63-68.

LASARTE, A. y ARRUÉ, M. Las bibliotecas de escuelas de música de Euskalherría: situación actual y futuro. En Actas del I Congreso de Escuelas de Música de Euskal Herria, 2000, p. 59-67.

MASSAULT, C. La formation professionelle des bibliothécaires musicaux en France. Bulletin des Bibliothèques de France, 2002, vol. 47, nº 2, p. 38-40.

MERLO, J.A. La formación del personal bibliotecario en centros de enseñanza musical. Jornadas sobre bibliotecas en conservatorios y escuelas de música. Madrid: Aedom, 1996, p. 275-284.

MERLO, J.A. Formación de los bibliotecarios musicales en España. En Actas del $18^{\circ}$ Congreso de la Asociación Internacional de Bibliotecas Musicales, Archivos y Centros de Documentación. Madrid: Aedom, 1999, p. 307-327.

MINISTERIO DE CULTURA. Encuesta de hábitos y prácticas culturales en España 2010-2011. Madrid: Ministerio de Cultura, 2011.

MIRANDA, F. La fonoteca. Madrid: Fundación Germán Sánchez Ruipérez, 1990.

MONTALT, M.L.; MOLINA, M.; FONTS, T.; PORTELL, N.; BUSQUIETS I LLEÓ, S.; SADURNÍ, M.; FERRÁN, J. y VEGA, L. La sección de música de la Biblioteca de Catalunya. Boletín DM, Aedom, 2010, año 14, p. 39-49. 
PLAZA, M.A. y CUENDE, M. La documentación musical en España ante el reto de las nuevas tecnologías: presente y ... ¿futuro? En Jadoc' 99: Segundas Jornadas Andaluzas de Documentación: nuevos mercados, nuevos usuarios. Granada: Asociación Andaluza de Documentalistas, 1999, p. 403-424.

POROILA, H. Public music libraries in Japan: facts and figures. Fontes Artis Musicae, 1989, vol. 36, n 2, p. 136-140.

RANERA, D. y CRESPO, L. Los cilindros sonoros en la BNE. Descripción y estudio. Criterios de conservación. Boletín DM, Aedom, 2010, año 14, p. 50-75.

REESER, D. Sixty years of public music libraries in the Netherlands. Fontes Artis Musicae, 1974, vol. 21, no 3, p. 92-96.

RODRIGO, A.M. Curso de catalogación de materiales especiales en formatos ISBD e IBERMARC: grabaciones sonoras, videograbaciones, recursos electrónicos en soporte óptico. Madrid: Estudio de técnicas documentales, 2007.

ROSE, I. Music in public libraries in Canada. Fontes Artis Musicae, 1987, vol. 34, nº 4, p. 184-187.

RUIZ, A. Guía para una fonoteca básica. Madrid: Ministerio de Cultura, 1985.

SETIÉN, E. Modelación matemática de bibliotecas en desarrollo. Investigación Bibliotecológica, 1998, vol. 12, nº 24, p. 41-48.

SKALICKY, J. Public music libraries in Hungary: their past and present. Fontes Artis Musicae, 1982, vol. 29, n 1-2, p. 13-15.

TEMES, J.L. Criterios de actuación de las administraciones públicas en materia de política musical. Alfoz, 1993, n $98-99$, p. 102-119.

TORRES, J. La documentación musical: luces y sombras. Boletín de la Fundación Juan March, 1991, n² 212, p. 3-18.

TORRES, J. Documentación musical: fondos y servicios en las Universidades públicas madrileñas. Revista General de Información y Documentación, 1995, vol. 5, nº 2, p. 163-185.

TORRES, J. El documento musical: ensayo de tipología. En: López Yepes, J. (ed.) Teoría, historia y metodología de las Ciencias de la Documentación (1975-2000). 1er. Congreso Universitario de Ciencias de la Documentación. Madrid: Facultad de Ciencias de la Información de la Universidad Complutense de Madrid, 2000, p. 743748. 